\title{
Estudios sobre los pueblos indígenas brasileños: una demografía con rostro humano
}

\author{
Studies on the Brazilian Indian Peoples: \\ a demography with a human face
}

\author{
María Mercedes Arias Valencia \\ Docente e investigadora, Maestría en Salud Colectiva \\ Facultad de Enfermería \\ Universidad de Antioquia, Colombia. \\ Carrera 43 , n. 23-53 apto. 202 \\ Medellín - Antioquia \\ mariamav@tone.udea.edu.co
}

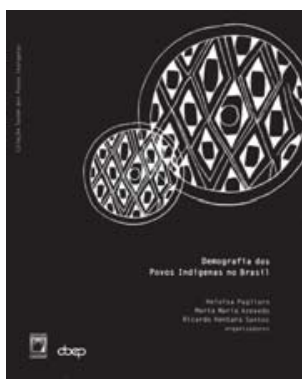

Pagliaro, Heloisa;

Azevedo, Marta Maria; Santos, Ricardo

Ventura (Org.).

Demografia dos povos

indígenas no Brasil. Rio

de Janeiro: Ed. Fiocruz;

Abep, 2005. 196p.
T a demografía de los pueblos indígenas es un objeto de conocimiento L complejo y multidimensional que tiene importancia, entre otros aspectos, por estudiar sociedades nativas ancestrales contemporáneas en transformación; su estudio es pertinente desde el punto de vista político, social y humano, por cuanto dichas sociedades, culturalmente diferenciadas, viven y se reproducen socialmente inmersas en sociedades nacionales que, en general, las han excluido y negado. Dada la pertinencia social, que no reside en el volumen, ni en el número absoluto sino en la complejidad de los procesos que viven y en la trascendencia de los cambios sociales, se comprende aún más la importancia de estos estudios.

El abordaje de dicho objeto requiere la concurrencia y cooperación de diversos saberes, entre las ciencias 'duras' - naturales -, y las ciencias 'blandas' - sociales -, en una interfase que dé cuenta, en forma integral, de la problemática de la sobrevivencia, de los cambios en su realidad sociocultural y de sus condiciones sociodemográficas.

La demografía de los pueblos indígenas es un campo de investigación no muy apetecido por los investigadores debido - entre otros - a la poca trascendencia relativa atribuida a las microsociedades; el desdén con que las ciencias 'duras' miran a los pequeños números puesto que dichas disciplinas surgen como herramientas para la macroeconomía, para estudiar sociedades urbanas e industriales con grandes números; el hecho de trabajar con datos de difícil obtención directa, de los que no se dispone registro sistemático o provienen de fuentes precarias, y con características de volatilidad - que explica parte de las oscilaciones de los indicadores -, además de las dificultades para el cálculo y la interpretación, hace necesario apropiar y aplicar cuidadosamente los procedimientos disponibles o idearse otros especialmente adecuados para esta situación.

Demografía dos povos indígenas no Brasil, organizado por Heloísa Pagliaro, Marta Maria Azevedo y Ricardo Ventura Santos, expone resultados de investigaciones que, de una manera crítica y reflexiva, hacen aportes teóricos y metodológicos sobre el tema de la dinámica demográfica en forma amplia, $y$, sobre sus componentes, la fecundidad, la nupcialidad, el comportamiento reproductivo, la morbilidad y la 
mortalidad, así como las herramientas para llevar a cabo los censos y los estudios específicos.

La publicación se sitúa en el campo de la demografía antropológica, la cual hace visible dichas sociedades por medio de la demografía y la estadística y les imprime especificidad sociocultural con las herramientas conceptuales y los sistemas de interpretación de las ciencias sociales. Los autores enfrentan los desafíos metodológicos haciendo un tratamiento riguroso de los datos a los que dotan de sentido creativamente, pues la demografía etnográfica estudia las tendencias de permanencia o de cambio de los patrones culturales en relación con la dinámica de la población y trata de imprimir un rostro humano para una demografía que no solo cuente personas, sino que, por el contrario, las personas cuenten.

Es mucho más valioso que los autores se interesen en la temática, dada la presencia numérica relativamente menor que tiene la población indígena en Brasil, respecto de otros países de América Latina. Los organizadores comienzan con el panorama crítico de los pueblos indígenas en Brasil y presentan su demografía desde el punto de vista diacrónico y sincrónico, resaltando los desdoblamientos recientes.

Marta Maria Azevedo estudia la nupcialidad de pueblos indígenas del alto río Negro y relaciona los patrones de matrimonio con los cambios demográficos que ocurrieron en dichos pueblos; da a conocer estrategias complejas de manejo de sus sistemas de parentesco y redes de intercambio que combinan la base lingüística con la de proximidad o lejanía de las localidades; analiza los pueblos como expulsores o receptores de mujeres que son, en última instancia, los objetos de dicho sistema de reciprocidad.

Cinco siglos después del contacto, tal como los autores Ricardo Ventura Santos, Nancy M. Flowers y Carlos E.A. Coimbra Jr. quieren expresar, aún estamos lejos de comprender a cabalidad la dinámica de disociación social y de despoblamiento que ocasionaron las enfermedades infecciosas en los pueblos indígenas y que hacen siempre pertinente y actual su análisis, dadas las penosas enseñanzas de la historia sobre su carácter emergente y reemergente y sobre las difíciles formas de control y erradicación. El trabajo de los autores enfoca los Xavante, pueblo indígena localizado en Mato Grosso. Dichas enfermedades, introducidas por los europeos, fueron y serán siempre exógenas, desconocidas y, tanto los pueblos indígenas como nuestra sociedad, mantenemos un vacío de estrategias para su erradicación y somos sorprendidos cada vez con su reemergencia y complejidad. Allí se sitúa la pertinencia de la relación entre epidemias, demografía y orden social.

Helósa Pagliaro, igual que otros autores y a partir del estudio de caso sobre los Kaiabi del Parque Indígena do Xingu, muestra los pueblos indígenas como sociedades que volvieron a crecer y pretenden buscar explicaciones que, aunque parciales, expliquen los cambios, a saber, el cese de conflictos, el aumento de la resistencia autóctona a los agentes infecciosos, la menor exposición a las epidemias, la acción exógena de control sobre las enfermedades infecciosas, la mejoría de las condiciones de salud, la garantía del territorio, la organización en instituciones que actúen en su propia defensa y el deseo y la decisión de crecer. 
Paulo Campanario avanza en la construcción de un modelo de estimación de indicadores de mortalidad, fecundidad y migración a partir de datos de composición de la población, lo cual constituye una esperanza. Su análisis compara resultados de las simulaciones con datos demográficos sobre los Kaiabi. Disponer de un modelo validado, confiable y accesible abre la posibilidad de ayuda para los investigadores que enfrentan un arduo trabajo con datos microdemográficos que requieren una adaptación rigurosa de las herramientas disponibles.

Cándido Procopio F. de Camargo, Carmen Junqueira y Heloísa Pagliaro analizan el comportamiento reproductivo de los Kamaiurá haciendo énfasis en las normas sociales que afectan las llamadas variables intermedias de la reproducción, que constituyen el componente sociológico de la fecundidad; las normas y valores sociales afectan de modo intencional la fecundidad y demuestran la plena racionalidad con que se entiende el proceso reproductivo.

A partir de investigación con los Sateré-Mawé, Pery Teixeira y Marília Brasil exponen la experiencia del censo participativo que constituye un reto; una de las conclusiones es el cambio en el comportamiento reproductivo de los residentes indígenas en áreas urbanas, quienes al poco tiempo en dichas áreas ya están comenzando a adquirir hábitos reproductivos de la población no indígena.

Nilza de Oliveira M. Pereira, Ricardo Ventura Santos y Marta Maria Azevedo dan a conocer un trabajo pionero de demografía antropológica a partir de los datos censales nacionales de Brasil, el cual es ejemplo de estudios posibles de realizar cuando hay un interés del Estado por avanzar con registros sistemáticos y apropiados. Los autores, además de hacer análisis demográfico, contribuyen con importantes reflexiones para el Instituto Brasileiro de Geografia e Estatística.

Para cerrar la publicación, Ricardo Ventura Santos, Heloísa Pagliaro y Marta Maria Azevedo dan a conocer una entrevista con John Early, autoridad mundial en el campo y autor de un libro clásico de demografía antropológica (sobre los Yanomami), quien hace un llamado a los estudios interdisciplinarios como una manera de encarar los desafíos de todo orden a que se enfrentan estos trabajos y esta publicación constituye un esfuerzo en ese sentido.

En resumen, Demografía dos povos indígenas no Brasil da a conocer distintos estudios demográficos aplicando las herramientas de la demografía en el estudio de temas tan importantes como las redes de intercambio en que se basan los sistemas de parentesco, las reglas de matrimonio, fecundidad y comportamiento reproductivo y la mortalidad, procesos vitales centrales para las sociedades, así como el sistema de asistencia en salud.

En todo el libro se apunta a la importante relación entre demografía y sociedad puesto que se vinculan los procesos vitales con las cuestiones históricas, políticas, económicas y de identidad cultural que van más allá de las estadísticas y hacen que la demografía sea verdaderamente demografía antropológica.

Con estos escritos es posible llegar a comprender la verdadera dimensión de los impactos del contacto y sus consecuencias para los pueblos indígenas, aún cinco siglos después, como también la verdadera 
complejidad de las circunstancias que los rodean y la riqueza cultural que les ha permitido tener conciencia y dominio sobre su homeostasis demográfica y les ha dado un anclaje que desafía el tiempo.

Desde el punto de vista metodológico, los aportes se caracterizan por el esfuerzo de aplicar, reflexivamente, sobre 'pequeños números', las herramientas de la demografía y por crear, recrear, validar y ajustar modelos, contrastándolos con datos reales y confiables; en una palabra, esfuerzos por mejorar las herramientas de que se dispone para abordar, en su verdadera complejidad, la realidad problemática de los pueblos indígenas.

Así pues, es notable que autores de distintas disciplinas hagan, desde la demografía antropológica, contribuciones rigurosas científicamente y pertinentes socialmente que hacen visible la cuestión indígena para la academia y, sobre todo, para el Estado y sus organismos, dando a conocer la dinámica demográfica y sus implicaciones históricas, económicas, políticas y culturales. Dichas contribuciones tienen aplicación en el diseño y seguimiento de políticas públicas económicas, educativas, de salud y desarrollo que consideren la especificidad cultural y la complejidad de dichas sociedades.

Los autores son investigadores con reconocida trayectoria en los ámbitos científico y académico que gozan de reconocimiento internacional por sus ejecutorias en el campo (mas no únicamente) de la demografía antropológica y cabe destacar que entre ellos se encuentran autoridades mundialmente reconocidas en este campo del saber. 